\title{
Sistema de extração seqüencial da solução na macro e microporosidade do solo
}

\author{
Thomas V. Gloaguen ${ }^{1}$, Francisco A. C. Pereira ${ }^{1}$, Roberta A. B. Gonçalves ${ }^{1} \&$ Vital da S. Paz ${ }^{1}$
}

\begin{abstract}
RESUMO
Existem várias técnicas de extração da solução no solo; entretanto, a proporção entre a água gravitacional e as retidas por forças capilares é geralmente desconhecida. Neste estudo se propôs desenvolver um sistema de extração seqüencial da solução no solo a fim de caracterizar a sua composição química em função da porosidade do solo. Construíram-se colunas com terra fina secada ao ar de um Cambissolo de textura argilo-siltosa. As colunas foram saturadas por $24 \mathrm{~h}$ com água destilada, logo após, a solução no solo foi coletada aplicando-se sucessivamente na base das colunas um vácuo de: $0 ;-13,3 ;-26,7 ;-40,0 ;-53,3 ;-66,6 \mathrm{kPa}$. Mediram-se o volume, o pH, a condutividade elétrica e as concentrações de $\mathrm{Na}^{+}, \mathrm{K}^{+}$e $\mathrm{NO}_{3}$. Houve boa correlação entre o vácuo aplicado e o potencial mátrico do solo medido por tensiometria (não linear, $\mathrm{r}^{2}=0,998$ ), validando o método proposto da extração seqüencial de solução. O estudo evidenciou importantes variações da composição química da solução nas diferentes porosidades do solo, com concentração iônica menor na macroporosidade, exceto para $\mathrm{K}^{+}$, demonstrando a necessidade de se uniformizar a metodologia de amostragem da solução do solo para evitar estimação incorreta da concentração de solutos no solo.
\end{abstract}

Palavras-chave: coluna de solo, solução no solo, porosidade, sódio, nitrato

\section{System of sequential extraction of solution in macro and microporosity of soil}

\begin{abstract}
Various methodologies for soil solution sampling are available, but the proportion between gravitational and capilar water is usually unknown. In this study, a sequential extraction system of soil water for determining its chemical composition as a function of the soil porosity is presented. Soil columns were filled by air-dried clay-loam Ultisol. The columns were saturated with distilled water for $24 \mathrm{~h}$, and then the soil solution was sampled at the base of the column by applying the suction equivalent to $0,13.3,26.7,40.0,53.3$ and $66.6 \mathrm{kPa}$. Volume, $\mathrm{pH}$, electrical conductivity, $\mathrm{Na}^{+}, \mathrm{K}^{+}$and $\mathrm{NO}_{3}{ }^{-}$ were measured in the solution. The high correlation (non linear; $\mathrm{r}^{2}=0.998$ ) between applied suction and soil matrix potencial, which was assessed by a tensiometer within the soil column, validated the proposed method for sequential soil solution extraction. lonic concentrations were highly variable in different soil pore sizes, with low concentrations in the soil macroporosity, except for $\mathrm{K}^{+}$, which demonstrated the necessity of standardizing the sampling methodology to avoid incorrect estimation of concentration of solute in soil.
\end{abstract}

Key words: soil column, soil solution, porosity, sodium, nitrate 


\section{INTRODUÇÃO}

A determinação da composição química da solução no solo é um procedimento amplamente utilizado para monitorar a fertirrigação (Silva et al., 2000; Perez \& Campos, 2003; Dias et al., 2005), estimar a lixiviação de poluentes (Laclau et al., 2003; Gloaguen et al., 2007) ou controlar a salinidade do solo (Dias et al., 2005). A fração aquosa no solo está retida no solo por forças matriciais (capilares e de adsorção), podendo então, com base nesse critério, ser separada em: (i) água gravitacional, livre, contida na macroporosidade do solo; (ii) água capilar, retida por forca capilar, contida nos microporos do solo; (iii) água adsorvida nos colóidos do solo, não disponível para as plantas. England (1974) notou uma composição química da solução muito diferente nos poros maiores daquela extraída dos poros finos do solo, devido à dissociação de cátions retidos na superfície das partículas coloidais. A difusão das espécies químicas é reduzida na água adsorvida nas partículas do solo, enquanto a água gravitacional sofre bastante os processos de dispersão e convecção no solo.

Várias técnicas de amostragem in situ são disponíveis, dependendo do objetivo do trabalho. Se o foco do estudo for a lixiviação de poluente, o mais importante será a recuperação da água gravitacional (De Rooij \& Stagnitti, 2000) e, neste caso, são utilizados lisímetros de tensão zero. Os lisímetros de tensão, também chamados extratores da solução no solo, permitem coletar uma solução intermediária, ou seja, uma mistura entre água de retenção e água livre, através da aplicação de uma sucção interna via bomba a vácuo (Barbee \& Brown, 1986; Grossman \& Udluft, 1991). O volume de solução coletada é fortemente afetado pelo material utilizado na confecção das cápsulas: cerâmica, teflon, nylon, vidro ou aço inoxidável, devido à porosidade específica de cada material (Grossman \& Udluft, 1991). Obviamente, o tipo e a quantidade de água coletada vão depender, também, do valor da sucção aplicada e do tempo de aplicação; no entanto, não se encontram valores padrões para esta técnica; além disso, a própria instalação dos lisímetros altera a quantidade de solução coletada (Surita et al., 2005). Os autores demonstraram que a instalação direta no solo, sem uso de areia lavada em volta da cápsula, permite a coleta de solução na porosidade mais fina do solo.

A extração da água capilar pode também ser feita com amostradores por capilaridade utilizando-se mecha de fibra de vidro, chamados amostradores de sucção passiva (Holder et al., 1991; Brandi-Dohrn et al., 1996). Esses amostradores são capazes de extrair água do solo com potencial de 0 a $-6,0 \mathrm{kPa}$, porém sem especificação mais precisa.

Em laboratório, a extração pode ser realizada mediante diversas técnicas, sendo que a mais conhecida e utilizada é a pasta de saturação (Rhoades, 1996); neste caso, a razão entre o volume de solo e o volume de água destilada adicionada é fixa. Neste método, o tempo de equilíbrio também é conhecido porém não há valor específico de sução e de tempo para extração da solução; pode-se esperar que a mudança desses parâmetros altera as características da solução coletada. A centrifugação é outra técnica utilizada em laboratório para se obter a solução mais retida no solo (Perez \& Campos, 2003; Gerard et al., 2003), mas nenhum protocolo satisfatório foi ainda desenvolvido. Perez \& Campos (2003) propuseram um valor de $2260 \mathrm{~g}$ durante $60 \mathrm{mn}$ para os solos brasileiros mas sem estabelecerem relação entre esta velocidade de centrifugação e o diâmetro dos poros mais finos alcançados. A extração por deslocamento miscível é outra técnica desenvolvida por Parker (1921) mas, além da baixa reprodutibilidade dos resultados, existem limitações, em termos de textura e de umidade de solos; por exemplo, raramente a técnica funciona em solos com umidade do ponto de murcha.

Diante das diversas técnicas de amostragem, pode-se constatar a falta de uniformização da amostragem; assim, a diferença de volume e de composição da solução coletada por técnicas diferentes sempre foi fonte de discussão. Os tipos de solo e de manejo do solo exercem grande influência na porosidade do solo e, conseqüentemente, na disponibilidade da solução no solo. Desta maneira, o uso idêntico de lisímetros de tensão, amostrador capilar ou centrífuga (tipo de instalação, intensidade e tempo de sucção ou rotação) em solos diferentes levará sistematicamente à coleta de solução em porosidades diferentes do solo. Landon et al. (1999) determinaram, através da análise de isótopos estáveis, que a solução obtida com lisímetros de tensão ou com amostrador capilar, em solo homogêneo e nas mesmas condições, contém uma fração entre 15 e 95\% de água gravitacional, sendo o restante água de retenção. Esta grande variabilidade reflete as dificuldades em se determinar o tipo de água coletada e impossibilita uma comparação entre métodos.

Objetivou-se, com este trabalho, o desenvolvimento de um sistema simples de extração seqüencial da solução no solo a partir de colunas elaboradas em laboratório, a fim de verificar a variabilidade da composição química da água no solo em função do diâmetro dos poros.

\section{MATERIAL E MÉTODOS}

Um protótipo de coluna de solo foi construído conforme ilustrado na Figura 1 e foi preenchido com terra fina secada ao ar (TSFA) de um Cambissolo Háplico Distrófico de textura argilo-siltosa $\left(80,405\right.$ e $515,0 \mathrm{~g} \mathrm{~kg}^{-1}$ de areia, silte e argila, camada $0-30 \mathrm{~cm}$ ), coletado no município de Simões Filho, BA. Durante a confecção da coluna, um tensiômetro foi inserido a $15 \mathrm{~cm}$ de profundidade.

O solo foi então saturado de forma ascendente com água destilada $\left(\mathrm{CE}_{\mathrm{a}}=0,007 \mathrm{dS} \mathrm{m}^{-1}\right)$ e permaneceu saturado por $24 \mathrm{~h}$. Em seguida, coletou-se a água gravitacional por drenagem. A água capilar foi coletada com auxílio de uma bomba elétrica de vácuo aplicando-se, sucessivamente, os vácuos: $-13,3 ;-26,7 ;-40,0 ;-53,3 ;-66,6 \mathrm{kPa}$ (diferença de $100 \mathrm{mmHg}$ entre os valores). Para cada valor de vácuo aplicado o potencial mátrico do solo $\left(\psi_{\mathrm{m}}\right)$ foi lido por meio de um tensímetro digital. $\mathrm{O}$ volume de cada fração foi medido por gravimetria para cálculo da umidade volumétrica do solo $(\theta)$ a partir da Eq. 1 e os pares de dados $\left(\psi_{m} ; \theta\right)$ foram ajustados no programa Table Curve 2D (Systat Software Inc.). 


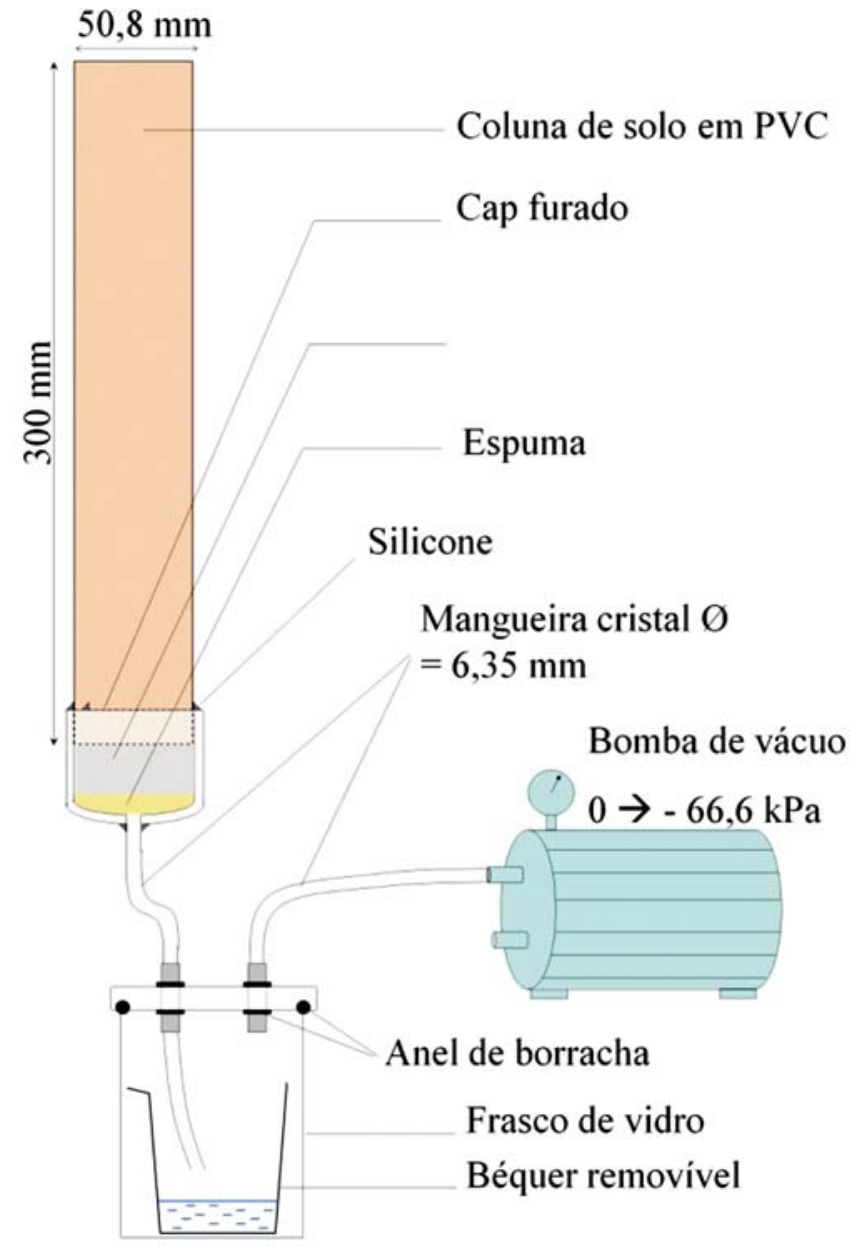

Figura 1. Detalhes da coluna de solo e da extração da solução no solo

$$
\theta=\frac{\mathrm{V}_{\mathrm{as}}-\mathrm{V}_{\mathrm{f}}}{\mathrm{m}_{\mathrm{s}}} \rho
$$

em que

$\mathrm{V}_{\mathrm{as}}$ - volume da água utilizada para saturar o solo, $\mathrm{m}^{3}$

$\mathrm{V}_{\mathrm{f}}$ - volume da fração coletado, $\mathrm{m}^{3}$

$\mathrm{m}_{\mathrm{s}}$ - massa do solo seco, $\mathrm{kg}$

$\rho$ - densidade aparente do solo, $\mathrm{kg} \mathrm{m}^{-3}$

$\mathrm{O}$ cálculo do raio médio de poros $\left(\mathrm{r}_{\mathrm{i}} \mathrm{em} \mu \mathrm{m}\right)$ correspon- dente ao potencial mátrico do solo $\left(\psi_{\mathrm{m}} \mathrm{em} \mathrm{kPa}\right)$ foi obtido com a lei de Jurin (Eq. 2).

$$
\mathrm{r}_{\mathrm{i}}=-\frac{2 \sigma \cos \alpha}{\rho_{\mathrm{a}} \mathrm{g}\left(0,102 \cdot \psi_{\mathrm{m}}\right)} 10^{6}
$$

em que

$\sigma$ - tensão superficial da água a $25{ }^{\circ} \mathrm{C}\left(0,071 \mathrm{~N} \mathrm{~m}^{-1}\right)$

$\alpha$ - ângulo de contato entre o líquido e o sólido $\left(0^{\circ}\right)$

$\rho_{\mathrm{a}}$ - densidade da água $\left(1000 \mathrm{~kg} \mathrm{~m}^{-3}\right)$

$\mathrm{g}$ - aceleração da gravidade $\left(9,81 \mathrm{~m} \mathrm{~s}^{-2}\right)$

A microporosidade, que corresponde ao volume de água retido no solo após aplicação de -6 kPa (EMBRAPA, 1997), inclui os poros inferior a um raio de $24 \mu \mathrm{m}$ se aplicarmos a Eq. 2. A macroporosidade equivale ao volume dos poros com raio superior a $24 \mu \mathrm{m}$. Para esse trabalho, aproximações foram necessários e determinou-se a macroporosidade drenável $\operatorname{MAPd}\left(\psi_{\mathrm{m}}>0 \mathrm{kPa}\right)$, macroporosidade não drenável MAPnd $\left(-6,5 \mathrm{kPa} \leq \psi_{\mathrm{m}}<0 \mathrm{kPa}\right)$ e a microporosidade MIP $\left(-13,1 \mathrm{kPa} \leq \psi_{\mathrm{m}}<-6,5 \mathrm{kPa}\right)$.

Num segundo tempo, nove colunas foram construídas conforme descrito acima. As colunas foram preenchidas até atingir densidade semelhante nas nove colunas $\left(1353 \pm 8 \mathrm{~kg} \mathrm{~m}^{-3}\right.$, $\mathrm{CV}=0,56 \%)$ correspondente à densidade do solo in situ (Tabela 1). Conseqüentemente, os volumes de macroporos e microporos obtidos foram muito parecidos, com variação de apenas 4,0 e 4,9\% (CV) para o volume de sólido e volume total de poros, respectivamente; da mesma forma, os valores de CV para as umidades do solo saturado e do solo após extração da solução a -66,6 kPa variam entre 5,0 e 7,2\%. Pode-se inferir, então, que as nove colunas são nove repetições; os valores de $\mathrm{CE}, \mathrm{pH}$, umidade e concentração iônica apresentados em seguida correspondem à média dos valores das nove colunas.

As colunas foram saturadas por $24 \mathrm{~h}$ com água destilada; em seguida, coletaram-se a água gravitacional e a água capilar aplicando-se, sucessivamente, vácuo de $0 ;-13,3 ;-26,7$; $-40,0 ;-53,3$ e $-66,6 \mathrm{kPa}$.

$\mathrm{O}$ calculo do raio a partir da Eq. 2 permitiu obter os seis intervalos de porosidade: (1) $\phi \geq 76,2 \mu \mathrm{m}$; (2) $44,6 \leq \phi<$ $76,2 \mu \mathrm{m}$; (3) 44,6 $\leq \phi<29,6 \mu \mathrm{m}$; (4) $29,6 \leq \phi<25,2 \mu \mathrm{m}$; (5) $25,2 \leq \phi<23,2 \mu \mathrm{m}$; (6) $23,2 \leq \phi<22,2 \mu \mathrm{m}$. Como o tamanho dos intervalos foi diferente, o volume coletado foi então dividido pela diferença entre os diâmetros mínimo e máximo de poro para cada intervalo a fim de obter o volume por

Tabela 1. Características físicas das colunas de solo

\begin{tabular}{ccccccrrrrr}
\hline Característica & Unidade & C1 & C2 & C3 & C4 & C5 & C6 & C7 & C8 & C9 \\
$\rho^{1}$ & $\mathrm{~kg} \mathrm{~m}^{-3}$ & 1.353 & 1.346 & 1.340 & 1.350 & 1.364 & 1.356 & 1.350 & 1.363 & 1.354 \\
$\rho_{\mathrm{s}}^{2}$ & $\mathrm{~kg} \mathrm{~m}^{-3}$ & 2.442 & 2.421 & 2.548 & 2.529 & 2.586 & 2.474 & 2.449 & 2.290 & 2.402 \\
$\mathrm{~V}_{\mathrm{s}}{ }^{3}$ & $\mathrm{~m} \mathrm{~m}^{-3}$ & 0,55 & 0,56 & 0,53 & 0,53 & 0,53 & 0,55 & 0,55 & 0,60 & 0,56 \\
$\mathrm{~V}_{\mathrm{P}}^{4}$ & $\mathrm{~m} \mathrm{~m}^{-3}$ & 0,45 & 0,44 & 0,47 & 0,47 & 0,47 & 0,45 & 0,45 & 0,40 & 0,44 \\
$\mathrm{~V}_{\text {MAPd }}{ }^{5}(\phi \geq 76,2 \mu \mathrm{m})$ & $\mathrm{m} \mathrm{m}^{-3}$ & 0,01 & 0,01 & 0,01 & 0,02 & 0,01 & 0,04 & 0,01 & 0,00 & 0,03 \\
$\mathrm{~V}_{\text {MAPnd }}{ }^{6}(44,6 \leq \phi<76,2 \mu \mathrm{m})$ & $\mathrm{m} \mathrm{m}^{-3}$ & 0,02 & 0,02 & 0,03 & 0,02 & 0,02 & 0,01 & 0,02 & 0,02 & 0,02 \\
$\mathrm{~V}_{\text {MIP }}{ }^{7}(\phi<44,6 \mu \mathrm{m})$ & $\mathrm{m} \mathrm{m}^{-3}$ & 0,42 & 0,41 & 0,43 & 0,33 & 0,44 & 0,40 & 0,42 & 0,38 & 0,40 \\
$\mathrm{U}_{\mathrm{s}}^{8}$ & $\mathrm{~kg} \mathrm{~kg}^{-1}$ & 0,329 & 0,330 & 0,354 & 0,345 & 0,346 & 0,334 & 0,333 & 0,297 & 0,322 \\
$\mathrm{U}_{\mathrm{F}}{ }^{9}$ & $\mathrm{~kg} \mathrm{~kg}^{-1}$ & 0,299 & 0,273 & 0,306 & 0,286 & 0,310 & 0,282 & 0,277 & 0,244 & 0,269 \\
\hline
\end{tabular}

$\mathrm{C1}$ - densidade aparente do solo; C2 - densidade de partículas; C3 - volume de sólidos do solo ( $\left.\mathrm{V}_{\mathrm{S}}=\mathrm{V}-\mathrm{V}_{\mathrm{P}}\right)$; $\mathrm{C} 4$ - volume de poros; $\mathrm{C5}$ - volume de macroporos drenáveis; C6 - volume de macropores não drenáveis; C7 - volume de microporos; C8 - umidade com base em peso do solo saturado; C9 - umidade com base em peso do solo após sução a -66,6 kPa 
unidade de poro (novo volume correspondente a um intervalo teórico de $1 \mu \mathrm{m}$ ); esse procedimento permite comparar os volumes das diferentes faixas. O comprimento médio dos poros Lp foi também calculado (Eq. 3):

$$
\mathrm{Lp}=\frac{\mathrm{Vss}}{\mathrm{Ap}}
$$

em que

Vss - volume da solução do solo coletada em $\mathrm{mm}^{3}$

Ap - área média do poro $=\pi \cdot r_{\mathrm{m}}{ }^{2}$, sendo $r_{m}$ o raio médio do intervalo de poros em $\mathrm{mm}$.

Para cada intervalo de poros, foram medidas $\mathrm{pH}, \mathrm{CE}$ e as concentrações de $\mathrm{Na}^{+}, \mathrm{K}^{+}$e $\mathrm{NO}_{3}^{-}$; escolheram-se esses íons pelo fato de apresentarem mais mobilidade na solução no solo (menos adsorção nos colóides do solo). Além disso, se calculou que esses íons representavam entre aproximadamente 30 e $85 \%$ do total de sais dissolvidos na solução extraída no solo (TDS $\approx$ CE.0,54; USSLS, 1954). Obtiveram-se as concentrações de $\mathrm{Na}^{+}, \mathrm{K}^{+}$e $\mathrm{NO}_{3}^{-}$com medidor de íon compacto Horiba ${ }^{\circledR}$ C-122, C-131 e C-141 calibrados, metodologia validada por Folegatti et al. (2005).

\section{RESULTADOS E DISCUSSÃO}

$\mathrm{O}$ vácuo aplicado e o potencial mátrico do solo foram bem correlacionados entre eles (Figura 2). Observa-se que o método é válido em uma faixa de potencial de 0 a $-13,1 \mathrm{kPa}$ (potencial mátrico máximo obtido); após a drenagem da água, o solo atingiu a umidade de capacidade de campo de $0,327 \mathrm{~kg} \mathrm{~kg}^{-1} \mathrm{e}$, ao final do ensaio, após a aplicação de um vácuo de $-66,6 \mathrm{kPa}$, a umidade do solo foi de $0,266 \mathrm{~kg} \mathrm{~kg}^{-1}$ (Tabela 2).

$\mathrm{O}$ diâmetro dos poros mais finos alcançados foi de 22,2 $\mu \mathrm{m}$ (Tabela 2), o que significa que se conseguiu extrair água dos microporos mais grossos, aproximadamente duas vezes mais finos do que os macroporos (cujo diâmetro mínimo é $50 \mu \mathrm{m}$ ). Não foi possível obter com este método a so-

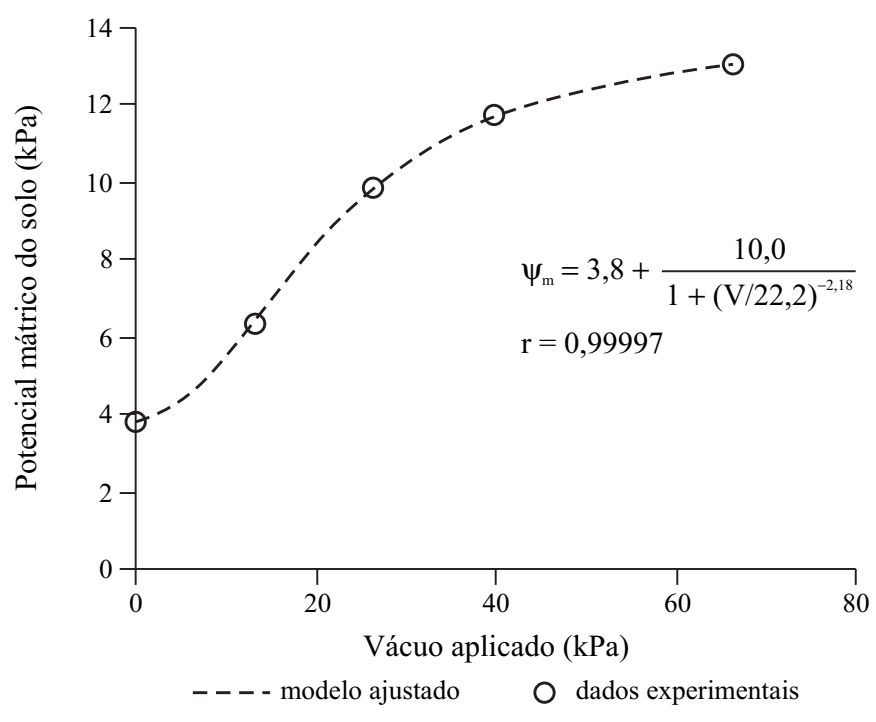

Figura 2. Potencial mátrico do solo $\left(\psi_{\mathrm{m}}\right)$ em função do vácuo aplicado $(\mathrm{V})$ na coluna para extração da solução no solo (valor absoluto) lução dos microporos mais finos com diâmetro de 22,2 até $0,2 \mu \mathrm{m}$, limite inferior proposto por Klein \& Libardi (2002).

Tabela 2. Vácuo aplicado $(V)$, potencial mátrico do solo $\left(\Psi_{\mathrm{m}}\right)$, volume de solução do solo coletada (Vss) e umidade com base em peso do solo (U)

\begin{tabular}{cccrcc}
\hline & $\mathbf{V}(\mathbf{k P a})$ & $\mathbf{\Psi m} \mathbf{( k P a )}$ & \multicolumn{2}{c}{$\left.\mathbf{V s s}(\mathbf{C V})^{\mathbf{4}} \mathbf{( c m}^{\mathbf{3}}\right)$} & $\left.\mathbf{U} \mathbf{~ ( k g ~ k g - 1 ~}^{-1}\right)$ \\
MAPd $^{1}$ & 0 & $-3,8$ & 19,1 & $(44,5)$ & 0,327 \\
MAPnd $^{2}$ & $-13,3$ & $-6,5$ & 12,3 & $(23,2)$ & 0,307 \\
MIP $^{3}$ & $-26,7$ & $-9,8$ & 12,2 & $(29,6)$ & 0,298 \\
MIP & $-40,0$ & $-11,5$ & 8,5 & $(34,6)$ & 0,283 \\
MIP & $-53,3$ & $-12,5$ & 7,2 & $(31,2)$ & 0,277 \\
MIP & $-66,6$ & $-13,1$ & 5,6 & $(25,2)$ & 0,266 \\
\hline
\end{tabular}

${ }^{1}$ macroporosidade drenável; ${ }^{2}$ macroporosidade não drenável; ${ }^{3}$ microporosidade $;{ }^{4}$ coeficiente de variação em \%

Com nove colunas, foi possível verificar a variabilidade do volume coletado, com base no coeficiente de variação (Tabela 2). O CV foi bastante similar para as diferentes classes de porosidade, com valores variando entre 23,2 e $44,5 \%$ (CV total $=31,4 \%$ ). No campo, a variabilidade do volume de solução coletada no solo com lisimetros de tensão por Gloaguen et al. (2007) foi de 66,5\%, sendo um quarto dos valores de CV maior do que $100 \%$ (dados do próprio autor, não publicados). A grande variabilidade do volume coletado é também freqüente em outras técnicas, tais como lisímetro de tensão zero (LTZ) onde pode ocorrer percolação preferencial ou amostrador por capilaridade passiva que funciona somente em uma faixa estreita de potencial mátrico (Boll et al., 1992).

\section{Características da porosidade do solo}

O volume de poros onde foi possível extrair solução totalizou somente $20 \%$ da porosidade total do solo, isto é, $80 \%$ da porosidade incluem poros com diâmetro inferior a 22,2 $\mu \mathrm{m}$. Observa-se, na Tabela 3, um decréscimo gradativo do volume de poros com a diminuição do diâmetro de poros. No entanto, como a variação do intervalo dos poros deve ser considerada, os volumes foram calculados por unidade de poro. Verifica-se então que o volume de poro aumenta à medida que diminui o diâmetro de poros, assim como o comprimento médio dos poros (Tabela 3).

Tabela 3. Características da porosidade do solo: intervalo de poros (I), diâmetro médio de poro ( $\phi)$, volume por unidade de poro (Vi), área total dos poros (Ap) e comprimento médio dos poros (Lp)

\begin{tabular}{|c|c|c|c|c|c|}
\hline & $\mathrm{I}(\mu \mathrm{m})$ & $\phi(\mu \mathrm{m})$ & Vi $\left(\mathrm{cm}^{3} \mu \mathrm{m}^{-1}\right)$ & $A p\left(m^{2}\right)$ & $\operatorname{Lp}(\mathrm{mm})$ \\
\hline $\mathrm{MAPd}^{1}$ & $>76,2$ & - & & & \\
\hline MAPnd ${ }^{2}$ & $44,6-76,2$ & 60,4 & 0,60 & $2,9.10^{-3}$ & $4,3.10^{3}$ \\
\hline MIP $^{3}$ & $29,6-44,6$ & 37,1 & 0,82 & $1,1.10^{-3}$ & $1,1.10^{4}$ \\
\hline MIP & $25,2-29,6$ & 27,4 & 2,77 & $5,9.10^{-4}$ & $1,4.10^{4}$ \\
\hline MIP & $23,2-25,2$ & 24,2 & 4,24 & $4,6.10^{-4}$ & $1,6.10^{4}$ \\
\hline MIP & $22,2-23,2$ & 22,7 & 7,15 & $4,0.10^{-4}$ & $1,4.10^{4}$ \\
\hline
\end{tabular}

\section{Composição química da solução no solo}

As concentrações de $\mathrm{Na}^{+}, \mathrm{K}^{+}$e $\mathrm{NO}_{3}{ }^{-}$foram calculadas após obtenção das curvas de calibração para cada medidor 
Tabela 4. Qualidade da análise da concentração de $\mathrm{Na}^{+}$(medidor Horiba ${ }^{\circledR}$ C-122) e de K+ (medidor Horiba ${ }^{\circledR}$ C-131) na solução no solo

\begin{tabular}{|c|c|c|c|c|}
\hline & \multicolumn{2}{|c|}{ C-122 } & \multicolumn{2}{|c|}{ C-131 } \\
\hline Concentração dos padrões $\left(\mathrm{mg} \mathrm{L}^{-1}\right)$ & $1-10$ & $50-1000$ & $1-10$ & $50-1000$ \\
\hline $\begin{array}{l}\text { Curva de calibração } \\
\mathrm{r}^{2}\end{array}$ & \multicolumn{2}{|c|}{ 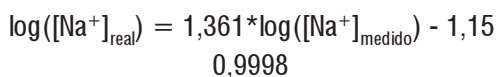 } & \multicolumn{2}{|c|}{$\begin{array}{c}{\left[\mathrm{K}^{+}\right]_{\text {real }}=1,040 *\left[\mathrm{~K}^{+}\right]_{\text {medido }}+2,29} \\
1,0000\end{array}$} \\
\hline Precisão ${ }^{1}(\%)$ & 4 & 1,5 & 2 & 1,3 \\
\hline Exatidão² (\%) & $270-600$ & $10-160$ & $20-60$ & $4-12$ \\
\hline
\end{tabular}

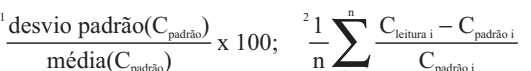

Horiba ${ }^{\circledR}$. A precisão das análises conferiu boa confiabilidade aos resultados (Tabela 4). Por outro lado, os medidores apresentaram grande variabilidade na exatidão (principalmente na leitura do $\mathrm{Na}^{+}$), demonstrado que o medidor não pode ser utilizado sem prévia calibração e análises periódicas de soluções padrões de concentração conhecida.

A solução no solo apresentou um $\mathrm{pH}$ menor na porosidade fina (Figura 3), o que pode ser explicado pela presença de íons hidrogênios na superfície das argilas (força de Van der Waals), e pela maior interação com os silicatos $\left(\mathrm{H}_{4} \mathrm{SiO}_{4}\right)$ que acidificam a solução (Gerard et al., 2003). Laclau et al. (2003) e Geibe et al. (2006) também acharam valores de $\mathrm{pH}$ menores na água capilar do que na água gravitacional. Em condições naturais (sequeiro), tal fenômeno pode ser esperado, mas o preparo do solo (calagem ou adubação) pode resultar em tendência inversa: soluções obtidas com amostradores de sucção passiva por Keith (1996) mostraram uma alcalinidade e $\mathrm{pH}$ maiores que as obtidas com lisímetros de tensão zero (LTZ). De qualquer forma, é importante ressaltar que sempre há diferença de $\mathrm{pH}$ com a porosidade, de acordo com as condições locais.

No presente estudo, o aumento de $\mathrm{H}^{+}$coincide com maior salinidade nos microporos, representado pelo valor crescente de CE ao longo da extração da solução no solo. Geibe et al. (2003) estudaram a composição química do solução no solo extraída por centrifugação, com cápsulas porosas e com LTZ e concluíram também que a concentração da maioria dos elementos monitorados era maior na solução centrifugada, ou seja, na solução nos microporos. Dentro dos sais monitorados no presente estudo, a concentração de nitrato foi particularmente alta, principalmente na microporosidade mais fina (Figura 3). Tal distribuição do nitrato foi também observada em campo, por Marques et al. (1996), em uma comparação da composição química da solução coletado no solo com cápsulas porosas (água capilar) e com LTZ (água gravitacional); entretanto, os autores não identificaram variação do $\mathrm{pH}$, contrariamente ao presente experimento, no qual o pH apresentou a variação mais nítida dentro dos parâmetros avaliados. Hendershot \& Courchesne (1991) também notaram uma concentração de $\mathrm{NO}_{3}{ }^{-}$maior na água capilar que na solução coletada com LTZ em campo. A adsorção de $\mathrm{NO}_{3}{ }^{-}$nos óxidos e hidróxidos carregados positivamente e que leva ao aumento da sua concentração na microporosidade, atenua a sua lixiviação até o lençol freático, no caso, por exemplo, da irrigação com águas residuárias ou de um excesso de adubo nitrogenado; além disso, o fato constitui uma reserva do nutriente para a planta.
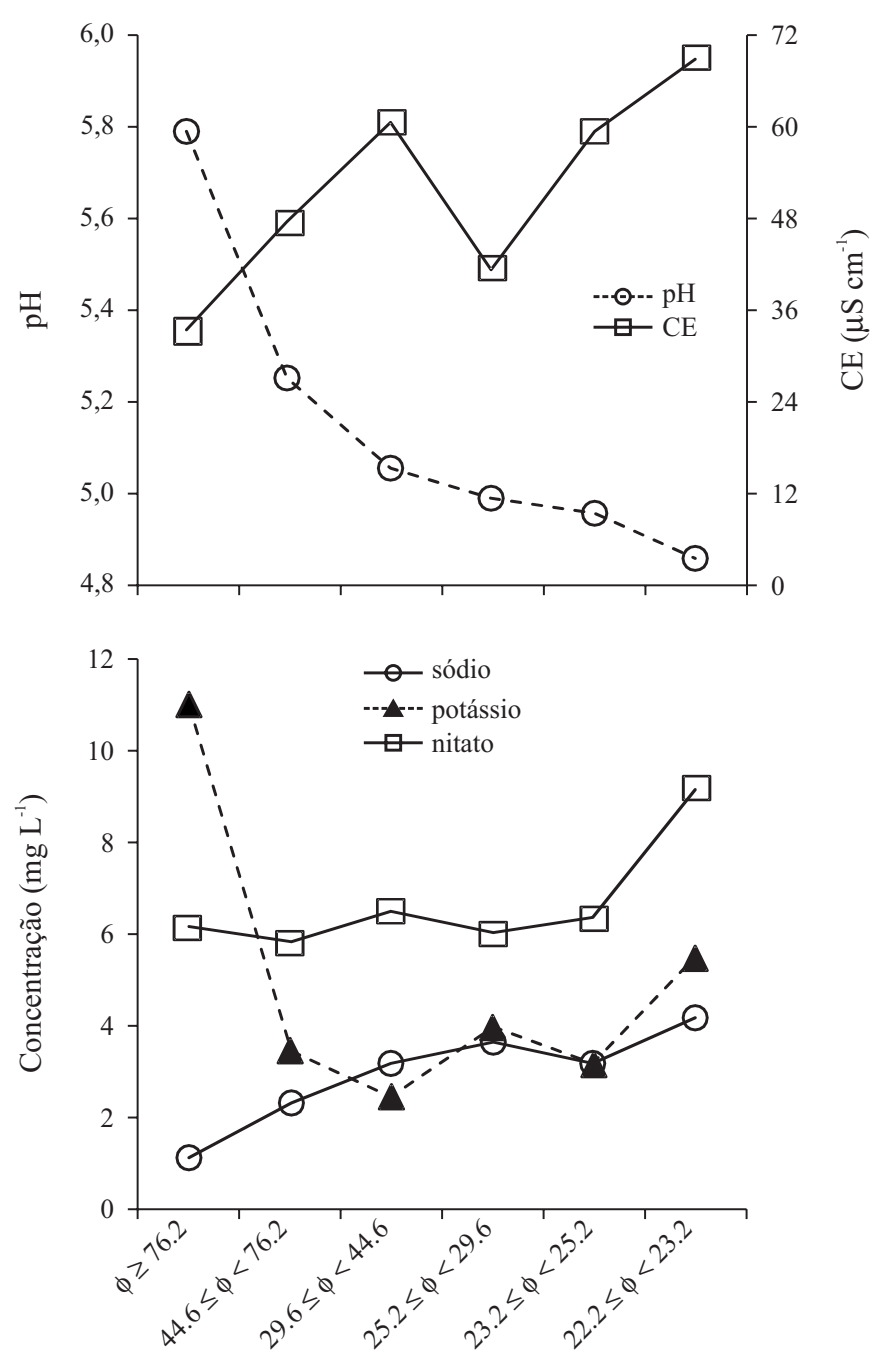

Figura 3. Condutividade elétrica, $\mathrm{pH}$ e concentração de $\mathrm{Na}^{+}, \mathrm{K}^{+}$e $\mathrm{NO}_{3}$. em função do diâmetro de poros

O mesmo comportamento foi observado para o sódio, isto é, um aumento da concentração deste elemento na porosidade fina (Figura 3), explicado pela troca iônica com o $\mathrm{K}^{+}$retido nas partículas de argila, pois a microporosidade inclui a porosidade intra-agregados. $\mathrm{O} \mathrm{K}^{+}$foi disponibilizado na solução e então lixiviado, o que explica o pico de concentração observado na macroporosidade (água gravitacional). Ressalta-se que os $80 \%$ de umidade retida no solo e que não puderam ser extraídos (Tabela 5) apresentavam, provavelmente, uma concentração iônica muito maior. Cuidado especial deve ser dirigido neste sentido, durante a irrigação com águas 
sódicas (concentração alta de $\mathrm{Na}^{+}$na porosidade fina promovendo grande impacto na expansão e dispersão das argilas). No campo, esses cátions parecem mais sensíveis às condições e manejos locais, pois Hendersot \& Courchesne (1991) não observaram variações de $\mathrm{Na}^{+}$ou $\mathrm{K}^{+}$entre a água gravitacional e a água capilar, enquanto Marques et al. (1996) notaram, independentemente, maior e menor concentração de $\mathrm{Na}^{+}$e $\mathrm{K}^{+}$, respectivamente, na microporosidade. Os resultados obtidos aqui são mais semelhantes aos de Ranger et al. (2001) que, após comparação da composição química da solução amostrada com centrifugação, cápsulas porosas e LTZ, afirmam que a qualidade da solução coletada variará se for aplicada, nas cápsulas porosas, uma tensão baixa (solução pouco concentrada) ou alta (solução muito concentrada).

De acordo com Marques et al. (1996), esses resultados confirmam que o monitoramento de nutrientes com lisímetros de tensão zero serve exclusivamente para determinação do balanço de entrada/saída de sais, haja vista que a composição química da solução obtida não é representativa daquela da solução no solo, enquanto amostradores (bem calibrados) de água capilar são necessários para obtenção do potencial nutritivo na porosidade fina do solo.

Os resultados estão sintetizados na Tabela 5: a maior salinidade ocorre na microporosidade, enquanto menores concentrações iônicas foram observadas na macroporosidade não drenada (umidade imediatamente abaixo da capacidade de campo). Outros trabalhos com outras classes de solos submetidos a diferentes manejos agrícolas serão necessários para descrever com precisão a distribuição dos sais na porosidade do solo.

Tabela 5. Porcentagem da porosidade total $(\% \mathrm{Vp})$, condutividade elétrica $\left(\mathrm{CE}, \mathrm{em} \mu \mathrm{S} \mathrm{cm}{ }^{-1}\right)$, pH e concentração em $\mathrm{Na}^{+}, \mathrm{K}^{+}$e NO${ }_{3}^{-}$(em mg L-1) da solução na macroporosidade e microporosidade de um Cambissolo Háplico Distrófico

\begin{tabular}{ccccccc}
\hline & $\% \mathbf{V}$ & $\mathbf{C E}$ & $\mathbf{p H}$ & $\mathbf{N a}^{+}$ & $\mathbf{K}^{+}$ & $\mathbf{N O}_{3}^{-}$ \\
MAPd $^{-}$ & 3 & 33,6 & 5,80 & 1,2 & 11,0 & 6,2 \\
MAPnd $^{2}$ & 4 & 47,7 & 5,26 & 2,3 & 3,5 & 5,8 \\
MIP $^{3}$ & 13 & 57,8 & 4,96 & 3,5 & 3,8 & 7,0 \\
\hline
\end{tabular}

${ }^{1}$ macroporosidade drenável $(\phi \geq 76,2 \mu \mathrm{m}) ;{ }^{2}$ macroporosidade não drenável $(44,6 \leq \phi<76,2 \mu \mathrm{m})$; ${ }^{3}$ microporosidade $(22,2 \leq \phi<44,6 \mu \mathrm{m})$

\section{CONCLUSÕES}

1. Demonstrou-se a eficiência da amostragem seqüencial da solução no solo aplicando-se vácuo em colunas preenchidas com solo; conseguiu-se identificar uma faixa de porosidade para cada solução coletada (alta correlação entre o vácuo aplicado e o potencial mátrico do solo).

2. A água gravitacional contida nos macroporos drenáveis tem menor concentração iônica (exceto $\mathrm{K}^{+}$) que a água capilar.

3. Isso demonstra a retenção das espécies químicas na água capilar do solo, oferecendo melhor disponibilidade de nutrientes para as plantas e limitando a lixiviação de poluentes na água gravitacional (pouco concentrada).

4. E necessário conhecer o valor da sucção aplicada na coleta da solução no solo para evitar subestimação ou supe- restimação da concentração iônica. Uma uniformização da metodologia é fundamental para se comparar dados de experimentos distintos.

\section{AGRADECIMENTOS}

Os autores agradecem à Fundação de Amparo à Pesquisa do Estado da Bahia - FAPESB - pela concessão da bolsa de pós-doutorado do primeiro autor.

\section{LITERATURA CITADA}

Barbee, G. C.; Brown, K. W. Comparison between suction and free-drainage soil solution samplers. Soil Science, v.141, p.149-154, 1986.

Boll, J.; Steenhuis, T. S.; Selker, J. S. Fiberglass wicks for sampling of water and solutes in the vadose zone. Soil Science Society of America Journal, v.56, p.701-707, 1992.

Brandi-Dohrn, F. M.; Dick, R. P.; Hess, M.; Selker, J. S. Field evaluation of passive capillary samplers. Soil Science Society of American Journal, v.60, p.1705-1713, 1996.

De Rooij, G. H.; Stagnitti, F. Spatial variability of solute leaching: experimental validation of a quantitative parameterization. Soil Science Society of American Journal, v.64, p.499-504, 2000.

Dias, N. da S.; Duarte, S. N.; Gheyi, H. R.; Medeiros, J. F. de; Soares, T. M. Manejo da fertirrigação e controle da salinidade do solo sob ambiente protegido, utilizando-se extratores de solução do solo. Revista Brasileira de Engenharia Agrícola e Ambiental, v.9, n.4, p.496-504, 2005.

EMBRAPA - Empresa Brasileira de Pesquisa Agropecuária. Centro Nacional de Pesquisa de Solos. Manual de métodos de análise de solo. 2.ed. Rio de Janeiro: EMBRAPA/CNPS, 1997. 212p.

England, C. B. Comments on "A technique using porous cups for water sampling at any depth in the unsatured zone" de Wood, W. W. Water Ressource Research, v.10, p.1049, 1974.

Folegatti, M. V.; Blanco, F. F.; Boaretto, R. M.; Boaretto, A. E. Calibration of cardy-ion meter to measure nutrient concentrations in soil solution and in plant sap. Scientia Agricola, v.62, n.1, p.8-11, 2005.

Geibe, C. E.; Danielsson, R.; Hees, P. A. W. van; Lundström, U. S. Comparison of soil solution chemistry sampled by centrifugation, two types of suction lysimeters and zero-tension lysimeters. Applied Geochemistry, v.21, p.2096-2111, 2006.

Gerard, F.; Ranger, J.; Ménétrier, C.; Bonnaud, P. Silicate weathering mechanisms determined using soil solutions held at high matric potential. Chemical Geology, v.202, p.443-460, 2003.

Gloaguen, T. V.; Forti, M. C.; Lucas, Y.; Montes, C. R.; Gonçalves, R. A. B.; Herpin, U.; Melfi, A. J. Soil solution chemistry of a Brazilian Oxisol irrigated with treated sewage effluent. Agricultural Water Management, v.88, p.119-131, 2007.

Grossmann, J.; Udluft, P. The extraction of soil water by the suctioncup method: A review. Soil Science, v.42, p.83-93, 1991.

Hendershot, W. H.; Courchesne, F. Comparison of soil solution chemistry in zero tension and ceramic-cup tension lysimeters. Journal of Soil Science, v.42, p.577-583, 1991. 
Holder, M.; Brown, K. W.; Thomas, J. C.; Zabcik, D.; Murray, H. E. Capillary-wick unsaturated zone soil pore water sampler. Soil Science Society of America Journal, v.55, p.1195-1202, 1991.

Keith, H. L. Principles of environmental sampling. Washington: American Chemical Society, 1996. 483p.

Klein, V. A.; Libardi, P. L. Densidade e distribuição do diâmetro dos poros de um Latossolo Vermelho, sob diferentes sistemas de uso e manejo. Revista Brasileira de Ciência do Solo, v.26, p.857-867, 2002.

Laclau, J. P.; Ranger, J.; Nzila, J. D.; Bouillet, J. P.; Deleporte, P. Nutrient cycling in a clonal stand of Eucalyptus and an adjacent savanna ecosystem in Congo. 2: Chemical composition of soil solution. Forest Ecology and Management, v.180, p.527-544, 2003.

Landon, M. K.; Delina, G. N.; Komorb, S. C.; Reganc, C. P. Comparison of the stable-isotopic composition of soil water collected from suction lysimeters, wick samplers, and cores in a sandy unsatured zone. Journal of Hydrology, v.224, n.1-2, p.45-54, 1999.

Marques, R.; Ranger, J.; Gelhaye, D.; Pollier, B.; Ponette, Q.; Goedert, O. Comparison of chemical composition of soil solutions collected by zero-tension plate lysimeters with those from ceramic-cup lysimeters in a forest soil. European Journal of Soil Science, v.47, p.407-417, 1996.
Parker, F. W. Methods of studying the concentration and composition of the soil solution. Soil Science, v.12, p.209-232, 1921.

Perez, D. V.; Campos, R. C. Solução do solo - importância e extração por centrifugação. Documentos da Empresa Brasileira de Pesquisa Agropecuária, v.48, p.22-38, 2003.

Ranger, J.; Marques, R.; Jussy, J. H. Forest soil dynamics during stand development assessed by lysimeter and centrifuge solutes. Forest Ecology and Management, v.144, p.129-145, 2001.

Rhoades, J. D. Salinity: Electrical conductivity and total dissolved solids. In: Sparks, D. L. (ed.) Methods of soil analysis: Chemicals methods. Madison: Soil Science Society of America/American Society of Agronomy, 1996. p.417-435.

Silva, E. F. F.; Anti, G. R.; Carmello, Q. A. C.; Duarte, S. N. Extratores de cápsulas porosas para o monitoramento da condutividade elétrica e do teor de potássio na solução de um solo. Scientia Agrícola, v.57, n.4, p.785-789, 2000.

Surita, C. A.; Gloaguen, T. V.; Montes, C. R.; Nogueira, S. F. Avaliação de desempenho de lisímetros de sucção para coleta de solução do solo. In: Congresso Brasileiro de Geologia de Engenharia e Ambiental, 41, 2005. Florianopolis. Resumos... Florianopolis: SBGEO, 2005. CD Rom.

USSLS - U.S. Salinity Laboratory Staff. Diagnosis and improvement of saline and alkali soils. Washington: U.S. Deptartment of Agriculture Handbook, 1954. 160p. 